Génét. Sél. Evol., 1986, 18 (2), 111-122

\title{
Caryotype de la Mouffette rayée, Mephitis mephitis
}

\author{
Fernande B. GENEST (1), P. MORISSET et R.P. PATENAUDE * \\ Département de Biologie, Faculté des Sciences et de Génie \\ Université Laval, Québec GIK 7P4, Canada \\ * Jardin zoologique de Québec, 8191, avenue du Zoo, Orsainville, \\ Québec GlG 4G4, Canada
}

\begin{abstract}
Résumé
Le caryotype de la Mouffette rayée [Mephitis mephitis (Schreber)] a été étudić dans des cultures fibroblastiques et lymphocytaires provenant de 4 mâles et de 6 femelles. Les observations en technique conventionnelle, en bandes $C$ et en bandes $G$, montrent un nombre diploïde de $2 n=50$ chromosomes, comprenant 22 paires de méta- submétacentriques et 2 paires d'acrocentriques. Le chromosome $X$ est un grand submétacentrique, dont l'identification précise n'est possible qu'en bandes $C$ ou $G$. Le chromosome $Y$, le plus petit élément du caryotype, est un acrocentrique. Un seul cas de polymorphisme a été rencontré : un individu possède un $\mathrm{Y}$ de plus grande taille que chez les autres mâles. Dans l'ensemble du caryotype, le marquage en bandes $C$ (hétérochromatine centromérique peu abondante, certains bras entièrement hétérochromatiques) et en bandes $G$ (plusieurs bras dépourvus de bandes) est très caractéristique.
\end{abstract}

Mots clés : Caryotype, mouffette rayée, Mephitis mephitis.

\section{Summary}

Karyotype of the striped skunk, Mephitis mephitis

The karyotype of the striped skunk [Mephitis mephitis (Schreber)] was studied in fibroblast and blood cultures from 4 males and 6 females. Observations of standard, C-banded, and G-banded karyotypes show a diploid number of $2 n=50$, with 22 pairs of meta- submetacentrics and 2 pairs of acrocentrics. The $X$ chromosome is a large submetacentric, which can be precisely identified only with $\mathrm{C}$ or $\mathrm{G}$ bands. The $\mathrm{Y}$, an acrocentric, is the smallest chromosome in the complement. One case of polymorphism was observed : one individual had a larger $Y$ than in other males. On the whole, the striped skunk's karyotype is quite characteristic by its C-banding (centromeric heterochromatin rare, some arms wholly heterochromatic) and G-banding (many arms without bands) patterns.

Key words : Karyotype, striped skunk, Mephitis mephitis.

(1) Adresse actuelle : Laboratoire de Cytogénétique, Département de Pathologie, Faculté de médecine, Université Laval, Québec G1K 7P4, Canada. 


\section{Introduction}

La famille des Mustélidés (ordre des Carnivores) présente un grand intérêt cytogénétique à cause de la variation importante du nombre et de la morphologie des chromosomes à l'intérieur de cette famille (Wurster \& BenirschKe, 1968). La Mouffette rayée [Mephitis mephitis (Schreber)] est un Mustélidé nord-américain dont les chromosomes ont été peu étudiés. Un nombre diploïde de $2 n=50$ a été signalé par FredGa $(1966,1967)$ et Hsu \& Benirschke (1967), mais aucun travail plus récent n'a été consacré au caryotype de cette espèce.

Cette étude avait pour buts d'étudier le caryotype d'un plus grand nombre d'individus et d'appliquer, en plus de la technique conventionnelle de coloration, les techniques de marquage en bandes $\mathrm{C}$ et $\mathrm{G}$, qui permettent une meilleure identification des paires de chromosomes.

\section{Matériel et méthodes}

Des mouffettes (Mephitis mephitis mephitis) (BANFIELD, 1974) des 2 sexes ont été étudiées, soit 4 mâles et 6 femelles. Toutes provenaient du Jardin zoologique de Québec. Des prélèvements de peau et de sang ont été effectués et ont permis d'établir des cultures fibroblastiques et lymphocytaires, respectivement. Les techniques de culture et de marquage ont été décrites antérieurement (GENEsT et al., 1979). Des renseignements supplémentaires sur l'utilisation de ces techniques et le choix du matériel sont présentés par GeNEST (1983).

La terminologie utilisée est celle proposée pour les chromosomes humains par le Système international pour la Nomenclature en Cytogénétique humaine (ISCN, 1978). Nous reconnaissons 3 types de chromosomes (acrocentriques, submétacentriques et métacentriques), classés en 2 groupes : les autosomes, formant 2 classes (les méta-submétacentriques et les acrocentriques), et les chromosomes sexuels. A l'intérieur de chaque groupe, les chromosomes sont ordonnés selon la taille décroissante des grands bras $(\mathrm{q})$.

\section{Résultats}

Les 2 types de cultures utilisées donnent des caryotypes superposables. Toutefois, les préparations obtenues à partir de cultures de peau sont de qualité supérieure. La dispersion cellulaire et chromosomique y est plus grande, sans nécessiter de traitement préalable. Aucune des techniques de coloration et de marquage n'a posé de problème particulier d'application. 
Les individus examinés ont un nombre diploïde de 50 chromosomes, comprenant 22 paires de métacentriques ou submétacentriques et 2 paires de petits acrocentriques (fig. 1 et 2). La formule gonosomique est de type XY. Le chromosome $X$ est un grand submétacentrique de la taille de la $3^{\mathrm{e}}$ ou $4^{\mathrm{e}}$ paire, alors que le chromosome $\mathrm{Y}$ est un acrocentrique minuscule et le plus petit élément du caryotype. Comme chez plusieurs autres Carnivores, le caryotype de la Mouffette possède une paire de marqueurs : la présence d'une constriction secondaire dans la région proximale des grands bras du chromosome $\mathrm{n}^{\circ} 7$, un submétacentrique de taille moyenne. Cette constriction, présente chez tous les individus, est visible sur presque toutes les préparations (fig. 1).

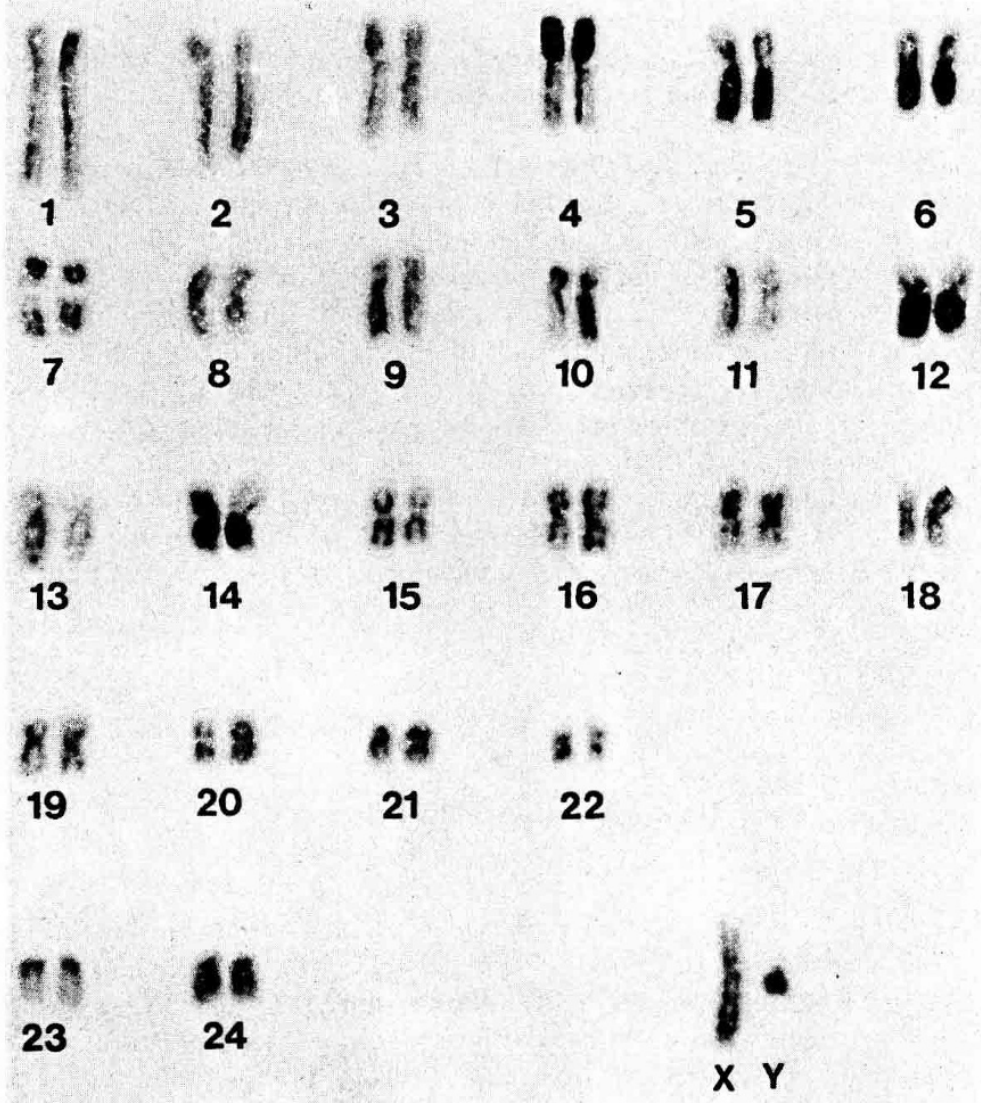

FIG. 1

Caryotype en bandes $C$ d'une mouffette rayée mâle, $2 n=50, X Y$. $C$-banded karyotype of a male striped skunk, $2 n=50, X Y$.

Les chromosomes $4,6,12$ et 14 ont un bras entièrement hétérochromatique. Le chromosome $\mathrm{Y}$ de cet individu était de taille plus grande que celui des 3 autres mâles étudiés.

Note that pairs $4,6,12$, and 14 have one wholly heterochromatic arm. The $Y$ chromosome of this individual was larger than in the 3 other males studied. 
Tous les individus étudiés présentent un caryotype semblable, sauf qu'un mâle possède dans toutes ses cellules un chromosome $\mathrm{Y}$ de taille plus grande que chez les 3 autres individus mâles. Ce chromosome, illustré dans le caryotype en bandes $\mathrm{C}$ (fig. 1), est identique dans les préparations provenant des 2 types de cultures. Aucune autre variation intraspécifique n’a été observée dans les caryotypes.

Le marquage du caryotype de la Mouffette en bandes $C$ (fig. 1, annexe A) se particularise surtout par la faible abondance d'hétérochromatine centromérique. Lorsque le centromère est souligné, c'est de façon à peine perceptible. D'autre part, certains chromosomes présentent des bras entièrement hétérochromatiques, avec un marquage de très forte intensité, notamment les paires $n^{08} 4,6,12$ et 14 (fig. 1). Le chromosome $X$ est très peu marqué, une seule bande de faible intensité apparaissant dans la partie distale du grand bras; il ne possède pas d'hétérochromatine centromérique de façon perceptible. Le chromosome $Y$, par contre, est entièrement hétérochromatique et facile à distinguer des plus petits chromosomes.

En bandes G (fig. 2, annexe B), le caryotype présente une caractéristique peu fréquente chez les Mammifères : l'absence totale ou presque de bandes sur un des bras ( $p$ ou $q$ ), notamment chez les paires $n^{0 s} 4,8,9,11,14$ et 18 . Lorsqu'il y a des bandes dans ces régions, elles ont une intensité très faible et sont à peine visibles. L'ensemble du caryotype possède toutefois une grande quantité de bandes positives, négatives ou variables (dont l'intensité varie d'une cellule à l'autre) et tous les homologues sont facilement identifiables. Le déroulement accentué de certaines paires chromosomiques met en évidence de fines bandes qui facilitent grandement l'identification. Les gonosomes se distinguent bien des autosomes. Le $\mathrm{X}$ présente, sur ses 2 bras, un marquage unique dans le caryotype par l'alternance et la disposition de ses bandes (fig. 2). Le chromosome $Y$ ne possède pas de marquage bien spécifique, étant presque entièrement variable ; seule une mince bande de faible intensité apparaît à sa partie distale.

\section{Discussion et conclusion}

Bien que le caryotype tel qu'observé par la méthode conventionnelle soit à peu près semblable à celui décrit par les auteurs antérieurs (FREDGA, 1966; Hsu \& BENIRSCHKE, 1967), l'utilisation des techniques de marquage en bandes $C$ et $G$ permet une bien meilleure identification des chromosomes de la Mouffette rayée. Les bandes $\mathrm{C}$, notamment, présentent un marquage très spécifique. De prime abord, le caryotype semble pauvre en hétérochromatine constitutive; cependant, l'absence presque complète de celle-ci au niveau centromérique, de même que le long des chromatides de la majorité des chromosomes, est compensée par la présence d'hétérochromatine constitutive sur la totalité des bras de certains chromosomes. Ce type de marquage en bandes $C$ est absent chez presque tous les mammifères dont le caryotype a été décrit. On le rencontre, toutefois, chez le Renard arctique (Alopex lagopus), dont 10 paires sur 24 ont un bras entièrement hétérochromatique (GENEST, 1983 ; Genest et al., en préparation). Cette particularité semble caractériser également plusieurs espèces de Mustélidés, notamment des espèces du genre Mustela. La 


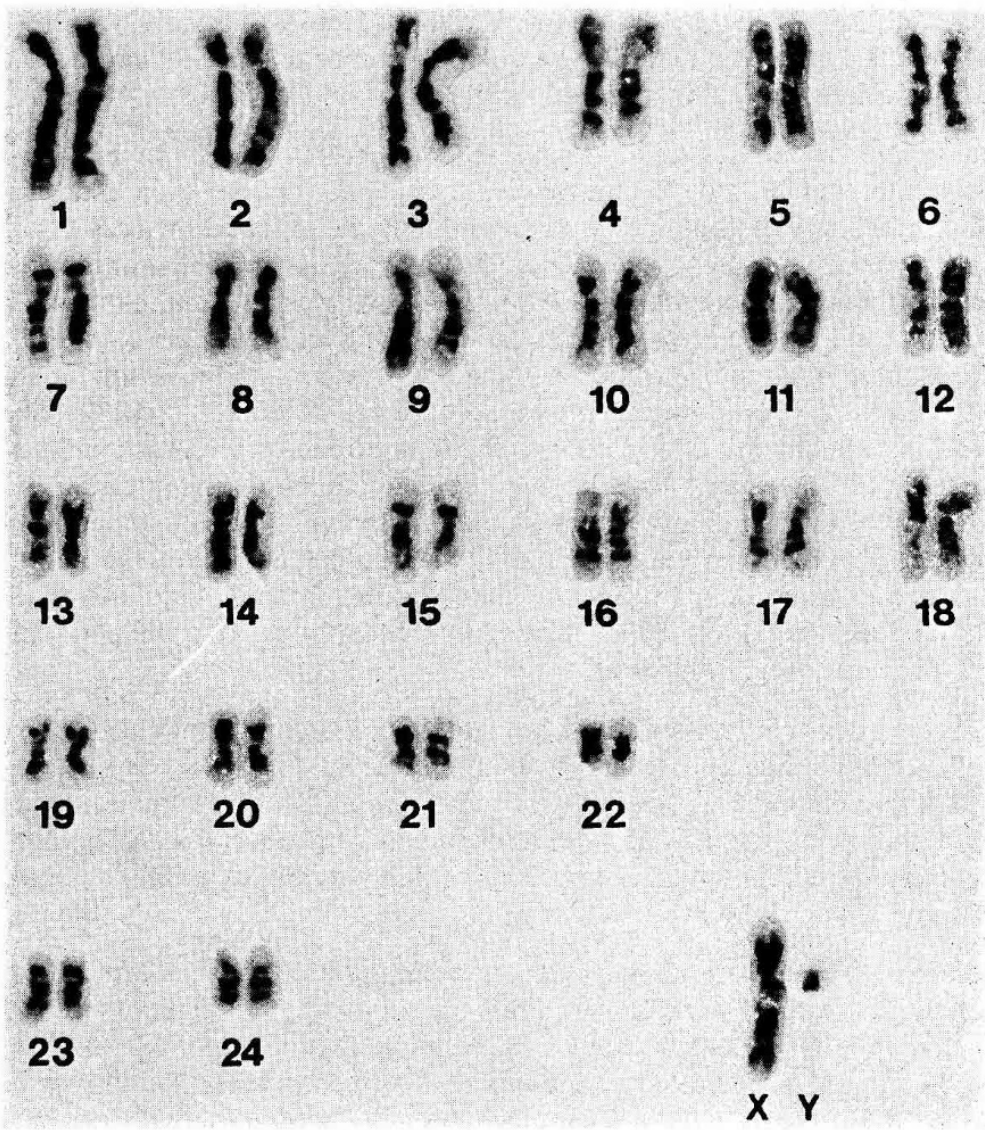

Fig. 2

Caryotype en bandes $G$ d'une mouffette rayée mâle, $2 n=50, X Y$. G-banded karyotype of a male striped skunk, $2 n=50, X Y$.

Les chromosomes $4,8,9,11,14$ et 18 présentent une absence totale ou presque de bandes sur l'un ou l'autre de leurs bras.

Note that chromosomes $4,8,9,11,14$, and 18 show no or almost no banding on one of their arms.

Belette commune (Mustela nivalis vulgaris) et la Belette pygmée (Mustela nivalis nivalis) ont, dans leur caryotype en bandes $C, 7$ bras entièrement hétérochromatiques (Grafodatsky et al., 1977 ; Mandahl \& Fredga, 1980), alors que le Putois européen (Mustela putorius) et le Putois des steppes (Mustela eversmanni) en ont respectivement 3 et 1 (Fredga \& Mandahl, 1973 ; Grafodatsky et al., 1977). Il semble y avoir des variations interspécifiques dans l'intensité de ce marquage, bien qu'il soit difficile d'apprécier ces variations, qui peuvent être le résultat de manipulations techniques 
différentes. L'utilisation de la technique de marquage en bandes $C$, trop souvent sous-estimée dans l'établissement des caryotypes, apporte dans le cas des Mustélidés autant d'informations discriminantes que celle des bandes $\mathbf{G}$.

Le marquage en bandes $\mathrm{G}$ a permis de constater l'absence de bandes marquées sur l'un ou l'autre des bras de 6 chromosomes. Ce phénomène, bien que présent chez d'autres Mammifères, n'est nulle part aussi fréquent que chez les Mustélidés. MaNDaHL \& Fredga (1980) remarquent que, chez la Belette, les bras chromosomiques peu marqués en bandes $\mathrm{G}$ ont tendance à être fortement marqués en bandes $\mathrm{C}$. Dans le cas de la Mouffette rayée, cette corrélation se vérifie pour les bras $4 p$ et $14 q$ seulement ; aucun autre bras peu marqué en bandes $G$ ne correspond à un bras fortement marqué en bandes C. Comme nous-mêmes, MaNdahl \& Fredga (1980) ont effectué leurs colorations en bandes $\mathrm{C}$ et $\mathrm{G}$ sur des préparations différentes; or, seul le traitement successif d'une même cellule par les 2 techniques en question pourrait, à notre avis, permettre d'établir clairement une telle correspondance entre marquage en bandes $\mathrm{C}$ et $\mathrm{G}$. Quant au chromosome $\mathrm{X}$, il présente un marquage comparable en tout point à celui observé chez beaucoup d'espèces de familles et d'ordres différents : Castoridés, Ursidés, Cervidés, Procyonidés, Canidés (Genest, 1983 ; Genest et al., 1979 et en préparation).

L'ensemble des caryotypes étudiés, notamment en bandes $C$, a permis de déterminer avec certitude que le chromosome $\mathrm{Y}$ de la Mouffette rayée est un acrocentrique. La présence chez un individu d'un $\mathrm{Y}$ de plus grande taille permet de confirmer sa nature acrocentrique. Comme aucune translocation avec d'autres chromosomes n'a pu être repérée chez cet individu, il semble exister un polymorphisme du chromosome $\mathrm{Y}$ chez la Mouffette rayée.

La présence d'une paire de chromosomes marqueurs chez la Mouffette n'étonne guère puisque les autres Mustélidés dont le caryotype est connu possèdent tous de une à trois paires de chromosomes marqueurs (Muldal, 1950; BenirschKe \& Low, 1966 ; Fredga, 1966, 1967 ; OMOdeo \& Renzoni, 1966 ; TAluKdar \& ManNa, 1966 ; Hsu \& BeNIRschKe, 1967 ; Wurster \& BeNIRschKe, 1967 ; Hsu \& BeNIRschKe, 1968). Bien que ces chromosomes soient présents chez toutes les espèces, chacune est caractérisée par des marqueurs bien spécifiques. Leur grande diversité à l'intérieur d'une même famille est interprétée par certains chercheurs (WURSTER \& BENIRSCHKE, 1968) comme un indice illustrant la tendance de ce groupe à accumuler des réarrangements chromosomiques. Ces derniers peuvent expliquer la grande variation du nombre diploïde $(2 n=30-64)$ chez les Mustélidés.

Reçu le 25 mars 1985.

Accepté le 27 novembre 1985

\section{Remerciements}

Nous remercions les autorités du Département de pathologie de la Faculté de médecine de l'Université Laval pour l'hospitalité accordée à F.B.G. dans leur Laboratoire de cytogénétique. La Direction du Jardin zoologique de Québec nous a permis la collecte des échantillons de peau et de sang. Cette étude a été réalisée grâce à des bourses d'études attribuées à F.B.G. par le Conseil national de recherches du Canada, le Ministère de l'Education du Québec et la Société zoologique de Québec. Ce travail a été en partie supporté par une subvention (A-5702) du Conseil de recherches en sciences naturelles et génie du Canada. 


\section{Références bibliographiques}

Banfield A.W.F., 1974. Les mammifères du Canada. 406 pp., Presses de l'Université Laval, Québec.

BenirschKe K., Low R.J., 1966. Chromosome studies on four carnivores. Mammal. Chrom. Newsl., 21, 148.

Fredga K., 1966. Chromosome studies in six species of Mustelidae and one of Procyonidae. Mammal. Chrom. Newsl., 21, 145.

Fredga K., 1967. Comparative chromosome studies of the family Mustelidae (Carnivora, Mammalia). Hereditas, 57, 295.

FredGa K., Mandahl N., 1973. Autosomal heterochromatin in some carnivores. Nobel Symp., 23, 104-117.

GENEST F.B., 1983. Etude chromosomique de quelques mammifères sauvages canadiens. 414 pp., Thèse de doctorat, Université Laval, Québec.

Genest F.B., Morisset P., Patenaude R.P., 1979. The chromosomes of the Canadian beaver, Castor canadensis. Can. J. Genet. Cytol., 21, 37-42.

Grafodatsky A.S., Ternovsky D.V., Isaenko A.A., Radzhabli S., 1977. Constitutive heterochromatin and DNA content in some Mustelids (Mustelidae, Carnivora). Genetika, 13, 2123-2128.

Hsu T.C., BentrschKe K., 1967. An atlas of mammalian chromosomes, Vol. 1, 1-50. Springer, Berlin-Heidelberg-New York.

Hsu T.C., BenirschKe K., 1968. An atlas of mammalian chromosomes, Vol. 2, 51-100. Springer, Berlin-Heidelberg-New York.

ISCN, 1978. An international system for human cytogenetic nomenclature. Cytogenet. Cell Genetics, 21, 313-404.

Mandahl N., Fredga K., 1980. A comparative chromosome study by means of G-, C-, and NOR-bandings of the weasel, the pygmy weasel and the stoat (Mustela, Carnivora, Mammalia). Hereditas, 93, 75-83.

Muldal S., 1950. A list of vertebrates observed at Bayfordbury, 1949/1950. John Innes Hort. Inst. 41th Ann. Rep., 39-41.

Omodeo P., Renzoni A., 1966. The karyotype of some Mustelidae. Caryologia, 19, 219-226.

Talukdar M., Manna G.K., 1966. Karyotypes of five carnivoran species from India. Mammal. Chrom. Newsl., 21, 151-153.

Wurster D.H., BenirschKe K., 1967. Chromosome number in thirty species of carnivores. Mammal. Chrom. Newsl., 8, 195.

Wurster D.H., BenirschKe K., 1968. Comparative cytogenetics studies in order Carnivora. Chromosoma (Berl.), 24, 336-382. 


\section{Annexe A}

Caractérisation du caryotype de la Mouffette rayée (Mephitis mephitis) en bandes C.

\begin{tabular}{|c|c|c|}
\hline $\mathrm{N}^{\circ}$ du chromosome & Région & Caractéristiques du marquage \\
\hline \multirow{2}{*}{\multicolumn{3}{|c|}{$\begin{array}{l}\text { Autosomes : } \\
\text { Métacentriques et } \\
\text { submétacentriques }\end{array}$}} \\
\hline & & \\
\hline 1 & Cen & Mince ; très faible intensité \\
\hline 2 & - & Non perceptible \\
\hline 3 & Cen & Très mince ; très faible intensité \\
\hline 4 & Cen $+p$ total & $\begin{array}{l}p \text { entièrement hétérochromatique; très forte } \\
\text { intensité }\end{array}$ \\
\hline 5 & - & Non perceptible \\
\hline 6 & Cen $+q$ total & $\begin{array}{l}\text { q entièrement hétérochromatique ; très forte } \\
\text { intensité }\end{array}$ \\
\hline 7 & - & Non perceptible \\
\hline 8 & Cen ? & Mince ; très faible intensité \\
\hline 9 & Cen & Très mince ; très faible intensité \\
\hline 10 & Cen $+p$ distal & Mince ; intensité moyenne \\
\hline 11 & Cen & Mince ; très faible intensité \\
\hline 12 & Cen $+q$ total & $\begin{array}{l}\text { q entièrement hétérochromatique; très forte } \\
\text { intensité }\end{array}$ \\
\hline 13 & Cen & Largeur moyenne ; intensité moyenne \\
\hline 14 & Cen $+q$ total & $\begin{array}{l}\text { q entièrement hétérochromatique ; intensité } \\
\text { moyenne }\end{array}$ \\
\hline 15 & Cen & Mince ; intensité moyenne \\
\hline 16 & Cen & Largeur moyenne; faible intensité \\
\hline 17 & $\begin{array}{l}\text { Cen }+q \text { proximal } \\
+p \text { total }\end{array}$ & $\begin{array}{l}\text { Largeur moyenne; intensité moyenne } \\
\text { Entièrement hétérochromatique }\end{array}$ \\
\hline 18 & - & Non perceptible \\
\hline 19 & Cen & Largeur moyenne ; intensité moyenne \\
\hline 20 & $\mathrm{p}+\mathrm{q}$ médian & Largeur moyenne; faible intensité \\
\hline 21 & Cen $+p$ proximal & Large ; intensité moyenne \\
\hline 22 & $\mathrm{q}$ distal & Large ; intensité moyenne \\
\hline \multicolumn{3}{|l|}{$\begin{array}{l}\text { Autosomes : } \\
\text { acrocentriques }\end{array}$} \\
\hline 23 & Cen & Large ; intensité moyenne \\
\hline 24 & Cen & Etroit ; faible intensité \\
\hline \multicolumn{3}{|l|}{$\begin{array}{l}\text { Chromosomes } \\
\text { sexuels }\end{array}$} \\
\hline $\mathrm{X}$ & $\begin{array}{l}\text { Cen } \\
\text { q distal }\end{array}$ & $\begin{array}{l}\text { Très faible } \\
\text { Mince ; très faible intensité }\end{array}$ \\
\hline Y & Cen $+q$ total & $\begin{array}{l}\text { Entièrement hétérochromatique ; très forte } \\
\text { intensité }\end{array}$ \\
\hline
\end{tabular}




\section{Annexe B}

Caractérisation du caryotype de la Mouffette rayée (Mephitis mephitis) en bandes G.

\begin{tabular}{|c|c|c|c|}
\hline $\mathrm{N}^{\circ}$ du chromosome & Bras & $\begin{array}{c}\text { Nombre } \\
\text { de régions }\end{array}$ & Repères \\
\hline \multicolumn{4}{|l|}{$\begin{array}{l}\text { Autosomes : } \\
\text { métacentriques et } \\
\text { submétacentriques }\end{array}$} \\
\hline \multirow[t]{2}{*}{1} & $\mathrm{p}$ & 2 & Bande médiane positive de forte intensité \\
\hline & $\mathbf{q}$ & 4 & $\begin{array}{l}\text { Large bande sous-proximale positive; large bande } \\
\text { sous-médiane positive; bande distale positive }\end{array}$ \\
\hline \multirow[t]{2}{*}{2} & $\mathrm{p}$ & 2 & Bande distale positive de forte intensité \\
\hline & q & 4 & $\begin{array}{l}\text { Bande proximale positive de forte intensité } \\
\text { large bande médiane variable ; bande pré-distale } \\
\text { positive }\end{array}$ \\
\hline \multirow[t]{2}{*}{3} & $\mathrm{p}$ & 2 & Bande pré-distale positive \\
\hline & $\mathrm{q}$ & 4 & $\begin{array}{l}\text { Bande proximale positive de forte intensité ; min- } \\
\text { ce bande sous-médiane positive ; bande pré-distale } \\
\text { positive }\end{array}$ \\
\hline \multirow[t]{2}{*}{4} & $\mathrm{p}$ & 2 & $\begin{array}{l}\text { Presque entièrement variable; très mince bande } \\
\text { médiane positive }\end{array}$ \\
\hline & $\mathrm{q}$ & 4 & $\begin{array}{l}\text { Bande sous-médiane négative ; large bande distale } \\
\text { variable }\end{array}$ \\
\hline \multirow[t]{2}{*}{5} & $\mathrm{p}$ & 2 & Bande proximale positive \\
\hline & $q$ & 3 & $\begin{array}{l}\text { Très large bande proximale positive; très large } \\
\text { bande distale positive }\end{array}$ \\
\hline \multirow[t]{2}{*}{6} & $\mathrm{p}$ & 2 & Bande médiane positive de forte intensité \\
\hline & $q$ & 3 & $\begin{array}{l}\text { Bande proximale positive; bande distale positive } \\
\text { de faible intensité }\end{array}$ \\
\hline \multirow[t]{2}{*}{7} & $\mathrm{p}$ & 2 & Bande médiane positive de forte intensité \\
\hline & $\mathrm{q}$ & 3 & $\begin{array}{l}\text { Bande proximale positive de forte intensité } \\
\text { bande distale positive }\end{array}$ \\
\hline \multirow[t]{2}{*}{8} & $\mathrm{p}$ & 1 & Entièrement variable \\
\hline & $\mathrm{q}$ & 3 & $\begin{array}{l}\text { Bande proximale positive de forte intensité ; large } \\
\text { bande distale variable }\end{array}$ \\
\hline \multirow[t]{2}{*}{9} & $\mathbf{p}$ & 2 & Mince bande médiane positive de faible intensité \\
\hline & $\mathrm{q}$ & 3 & $\begin{array}{l}\text { Très large bande médiane positive de forte } \\
\text { intensité; mince bande pré-distale positive }\end{array}$ \\
\hline \multirow[t]{2}{*}{10} & $\mathrm{p}$ & 2 & Large bande proximale positive \\
\hline & $\mathbf{q}$ & 4 & $\begin{array}{l}\text { Bande proximale positive suivie d'une bande né- } \\
\text { gative ; large bande distale variable }\end{array}$ \\
\hline
\end{tabular}


Annexe B (suite)

\begin{tabular}{|c|c|c|c|}
\hline $\mathrm{N}^{\circ}$ du chromosome & Bras & $\begin{array}{c}\text { Nombre } \\
\text { de régions }\end{array}$ & Repères \\
\hline \multirow[t]{2}{*}{11} & $\mathrm{p}$ & 3 & $\begin{array}{l}\text { Large bande proximale négative; bande médiane } \\
\text { positive }\end{array}$ \\
\hline & $\mathbf{q}$ & 3 & $\begin{array}{l}\text { Bande proximale positive; très large bande dis- } \\
\text { tale variable }\end{array}$ \\
\hline \multirow[t]{2}{*}{12} & $\mathrm{p}$ & 3 & $\begin{array}{l}\text { Bande proximale positive; bande pré-distale po- } \\
\text { sitive }\end{array}$ \\
\hline & q & 3 & $\begin{array}{l}\text { Bande sous-proximale positive de forte intensité; } \\
\text { bande distale variable }\end{array}$ \\
\hline \multirow[t]{2}{*}{13} & p & 2 & Bande proximale positive \\
\hline & q & 4 & $\begin{array}{l}\text { Bande proximale positive de forte intensité suivie } \\
\text { d'une bande médiane négative; large bande dis- } \\
\text { tale variable }\end{array}$ \\
\hline \multirow[t]{2}{*}{14} & p & 2 & Bande médiane positive de forte intensité \\
\hline & q & 3 & $\begin{array}{l}\text { Presque entièrement variable; bande médiane } \\
\text { positive de faible intensité }\end{array}$ \\
\hline \multirow[t]{2}{*}{15} & $\mathrm{p}$ & 2 & Large bande proximale positive \\
\hline & $\mathrm{q}$ & 3 & $\begin{array}{l}\text { Bande médiane positive de forte intensité; très } \\
\text { large bande distale variable }\end{array}$ \\
\hline \multirow[t]{2}{*}{16} & $\mathrm{p}$ & 2 & Bande médiane positive \\
\hline & $q$ & 3 & $\begin{array}{l}\text { Bande médiane positive d'intensité moyenne sui- } \\
\text { vie d'une large bande pré-distale variable; bande } \\
\text { distale positive d'intensité moyenne }\end{array}$ \\
\hline \multirow[t]{2}{*}{17} & $\mathrm{p}$ & 3 & $\begin{array}{l}\text { Bande proximale positive de forte intensité ; très } \\
\text { large bande distale variable }\end{array}$ \\
\hline & q & 3 & $\begin{array}{l}\text { Bande proximale positive de forte intensité; } \\
\text { bande médiane positive }\end{array}$ \\
\hline \multirow[t]{2}{*}{18} & $\mathbf{p}$ & 2 & Bande distale positive de faible intensité \\
\hline & q & 3 & $\begin{array}{l}\text { Bande proximale positive de faible intensité; } \\
\text { large bande distale variable }\end{array}$ \\
\hline \multirow[t]{2}{*}{19} & $\mathrm{p}$ & 2 & Bande distale positive de forte intensité \\
\hline & q & 2 & Très large bande distale positive de forte intensité \\
\hline \multirow[t]{2}{*}{20} & $\mathrm{p}$ & 3 & $\begin{array}{l}\text { Bande proximale variable; mince bande distale } \\
\text { positive }\end{array}$ \\
\hline & q & 3 & $\begin{array}{l}\text { Bande proximale positive de faible intensité; } \\
\text { large bande distale variable }\end{array}$ \\
\hline \multirow[t]{2}{*}{21} & $\mathrm{p}$ & 2 & Bande proximale positive d'intensité moyenne \\
\hline & $q$ & 3 & $\begin{array}{l}\text { Bande proximale positive d'intensité moyenne; } \\
\text { large bande distale négative }\end{array}$ \\
\hline
\end{tabular}


Annexe B (suite)

\begin{tabular}{|c|c|c|c|}
\hline $\mathrm{N}^{\circ}$ du chromosome & Bras & $\begin{array}{c}\text { Nombre } \\
\text { de régions }\end{array}$ & Repères \\
\hline 22 & $\begin{array}{l}\mathrm{p} \\
\mathrm{q}\end{array}$ & $\begin{array}{l}1 \\
2\end{array}$ & $\begin{array}{l}\text { Large bande variable } \\
\text { Bande proximale positive d'intensité moyenne }\end{array}$ \\
\hline \multicolumn{4}{|l|}{$\begin{array}{l}\text { Autosomes : } \\
\text { acrocentriques }\end{array}$} \\
\hline 23 & $q$ & 4 & $\begin{array}{l}\text { Bande proximale positive de forte intensité ; large } \\
\text { bande médiane positive de faible intensité ; bande } \\
\text { distale positive de faible intensité }\end{array}$ \\
\hline 24 & $q$ & 3 & $\begin{array}{l}\text { Bande proximale positive de faible intensité } \\
\text { bande médiane positive de forte intensité }\end{array}$ \\
\hline \multicolumn{4}{|l|}{$\begin{array}{l}\text { Chromosomes } \\
\text { sexuels }\end{array}$} \\
\hline$x$ & $\mathrm{p}$ & 2 & $\begin{array}{l}\text { Très large bande médiane positive de forte } \\
\text { intensité }\end{array}$ \\
\hline & $\mathrm{q}$ & 4 & $\begin{array}{l}\text { Bande proximale positive d'intensité moyenne } \\
\text { suivie d'une large bande pré-médiane négative; } \\
\text { très large bande médiane positive de forte inten- } \\
\text { sité ; bande pré-distale positive }\end{array}$ \\
\hline $\mathrm{Y}$ & $q$ & 2 & $\begin{array}{l}\text { Très large bande variable ; très mince bande posi- } \\
\text { tive de faible intensité à la partie distale }\end{array}$ \\
\hline
\end{tabular}

